\title{
Cross-Layer Optimization for Efficient Delivery of Scalable Video over WiMAX
}

\author{
Lung-Jen Wang ${ }^{1, a^{*}}$, Chiung-Yun Chang ${ }^{2, b}$ and Jen-Yi Huang ${ }^{3, c}$ \\ ${ }^{1,3}$ Department of Computer Science \& Information Engineering, \\ National Pingtung University, Taiwan \\ ${ }^{2}$ Information Technology Department, Yuanta Bank, Taiwan \\ a ljwang@mail.nptu.edu.tw, ${ }^{\mathrm{b}}$ changmp6@yuanta.com, ${ }^{\mathrm{C}}$ huangjy@mail.nptu.edu.tw \\ * the corresponding author
}

Keywords: Cross-layer optimization; scalable video; WiMAX; packet classifier; queue allocation.

\begin{abstract}
Video delivery application over wireless network has become increasingly important in recent years. For achieving more flexible video streams transmission, the scalable extension of the H.264/AVC standard has been developed, as well as which is combined with temporal, spatial and quality scalabilities to adapt efficiently the wireless bandwidth resource. In this paper, a cross-layer optimization for robust H.264 scalable video delivery over WiMAX network is proposed. In order to obtain a better delivery performance, the proposed method is used the hierarchical B-picture approach to classify video packets into different priorities. In the cross-layer optimization, the scalable video and packet classifier are proposed at the application layer, and the packet priority and queue allocation are used at the MAC layer. Finally, it is shown by NS2 simulations that the proposed method provides more stable wireless bandwidth and also achieves a better quality of the video streaming delivery.
\end{abstract}

\section{Introduction}

The technology of multimedia application over wireless network, including video streaming, is growing faster in recent years. This technology increases the convenience and entertainment of the distant communication. The best-effort service supporting wireless network technology, however, can no longer achieve such requirement of the video streaming as the sensibility to delay time, fluctuations in bandwidth, and packet drop. A WiMAX (Worldwide Interoperability for Microwave Access) technology which is based on IEEE 802.16 standard is intended to support the video streaming [8]-[9]. To achieve the most efficient performance of video streaming delivery, many cross-layer mechanisms which are used the cooperation between APP (application) layer and lower layers during the transmission session are proposed [1][6][7][10]-[16].

In order to provide cross-layer optimization, a suitable architecture should be designed for video delivery over wireless networks [15]. Most previous studies on cross-layer optimizations focus on PHY (physical) and MAC (Medium Access Control) layer interactions and do not explicitly consider the APP layer performance. Basically, a cross-layer design involved feedbacks received from other layer to optimize the parameters in the current layer so that optimum performance for video delivery is achieved [10].

To achieve more flexibility video streams in multimedia transmission, the Joint Video Team (JVT) is also developed the scalable extension of the H.264/AVC standard, known as H.264/SVC [3][10]. It is combined with temporal, spatial and quality scalabilities to obtain a better performance and more adaptation of the efficient wireless bandwidth resource. This paper is to present a cross-layer optimization for delivering H.264 scalable video over WiMAX network. To obtain a better performance, the hierarchical B-picture approach [2] is used to classify video packets into different priorities. In addition, the drop video packets are based on the queue utilizations. That is, the cross-layer scheme proposes the packet classifier at the APP layer and the queue allocation at the MAC layer to achieve more stable and available queues for the scalable video delivery. Finally, it is shown by NS2 simulations that the proposed method can yield a better performance for transmission loss rate, image quality and packets throughput over WiMAX networks. 


\section{Our Previous Work}

The WiMAX system is composed of at least one base station (BS) and more than one subscriber stations (SSs). Two modes in the system: (1) point-to-multipoint mode which a BS controls all communications, and (2) mesh mode which direct communications are used between SSs. Since there is possibility that the wireless channel becomes unavailable, therefore cross-layer interactions between the PHY and MAC layers are needed so that the limited wireless resource could be optimally used while satisfying the video delivery requirements. In [16], a cross-layer framework is proposed to integrate layer 3 and layer 2 and also a remapping scheme is used for a better buffer utilization allowing a low frame dropping ratio. In this paper, a cross-layer architecture between APP and MAC layers is proposed. Optimization is performed at the BS which delivers video data to SSs.

The H.264/SVC technique [13] which is used in this paper. It facilitates efficient video transmissions. With the SVC scheme, a single video sequence is encoded to generate a base and several enhancement layers. In the encoder, the SVC combines with temporal, spatial and quality (SNR) scalabilities in a single video sequence (bit stream) by the use of several video layers, which consist of a VCL (video coding layer) and a NAL (network abstraction layer). The VCL uses a block-based hybrid coding structure and provides various scalabilities. Hierarchical prediction structure supports temporal scalability. Inter-layer motion prediction, inter-layer residual prediction and inter-layer intra-prediction are used to provide spatial scalability. In addition, the NAL is used to format the encoded video streams into NAL units and provide header information. For more details on SVC, please refer to [13].

Our previous work on the cross-layer optimization [10]-[11] and enhances it even further. It follows from [10]-[11], let $S_{A P P}, S_{N A T}, S_{M A C}$, and $S_{P H Y}$ denote the set of parameters available at the APP, NAT (Network and Transport), MAC, and PHY layers, respectively. The joint cross-layer strategy $S$ is defined as $S=\left\{S_{A P P}, S_{N A T}, S_{M A C}, S_{P H Y}\right\}$. Then, the cross-layer architecture is to find the optimal parameter set

$$
S^{o p t}(x)=\arg \max _{S} Q(S(x)) \text { such that } \operatorname{rate}(S(x)) \leq R_{b} \text { and } \operatorname{delay}(S(x)) \leq D_{\max },
$$

where the instantaneous channel condition $x$, the video quality $Q$ is an estimated PSNR (peak signal-to-noise ratio), the value $R_{b}$ is the available transmission bit rate while the bound $D_{\max }$ (maximum accepted delay).

Nevertheless, the best solution for cross-layer architecture still depends on the application requirements. By the close communication on this architecture, all layers in the protocol can reflect the changing environment on time and achieve the goal of efficient system resource management.

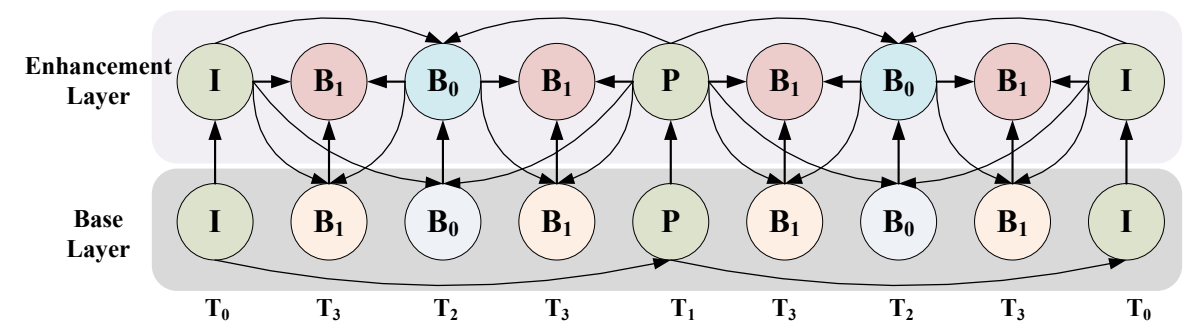

Fig. 1. The hierarchical B-picture approach.

\section{Proposed Cross-Layer Optimization}

Hierarchical B-picture Approach. Following the SVC structure, a scalable video bit-stream composes of quality and temporal layers. The group of picture (GOP) size is $f$ frames at a maximum frame rate of $r$ frames/sec. Temporal enhancement frames are encoded as hierarchical bi-predictively coded (B) pictures, as shown in Fig. 1. While B-pictures introduce structural delay due to out-of-order frame decoding, they provide a higher coding efficiency. Furthermore, the video bit-stream is divided into I, P and B-slices by the VCL layer. In the experiment of this paper, the size of a GOP is 8 pictures with picture pattern (No. of $\mathrm{I}=1$, No. of $\mathrm{P}=2$, and No. of $\mathrm{B}=3$ ), and the sequence of video coding is $\mathrm{I}, \mathrm{P}$, 
$\mathrm{B}_{0}, \mathrm{~B}_{1}$, and $\mathrm{B}_{1}$. In addition, temporal layer consists of four layers: I-frame is temporal layer $0\left(\mathrm{~T}_{0}\right)$, P-frame is temporal layer $1\left(T_{1}\right)$, and B-frames are temporal layer 2 and $3\left(T_{2}\right.$ and $\left.T_{3}\right)$, respectively. [2][13]

Queue Utilization. In WiMAX, the MAC layer uses some queues to manage the video packets. Furthermore, MAC maps the video packets in only queue management neglecting the packet priority and queue congestion it may loss important video packets that degrades the video quality [5]. Therefore, we proposes a queue utilization, as shown in Fig. 2, which is an adaptive queue approach to adjust the video packet transmission between APP and MAC layers via cross-layer architecture. In other words, the APP layer sends the video packet to the WiMAX network based on the hierarchical B-picture approach which the SVC video packets (I, P, $\mathrm{B}_{0}$, and $\mathrm{B}_{1}$ ) and the packet types are used. In the MAC layer, the video packets are passed to the PHY layer, which is based on the packet priority and the queue utilization, i.e., the MAC layer performs the dynamic queue allocation for appropriate video packet.

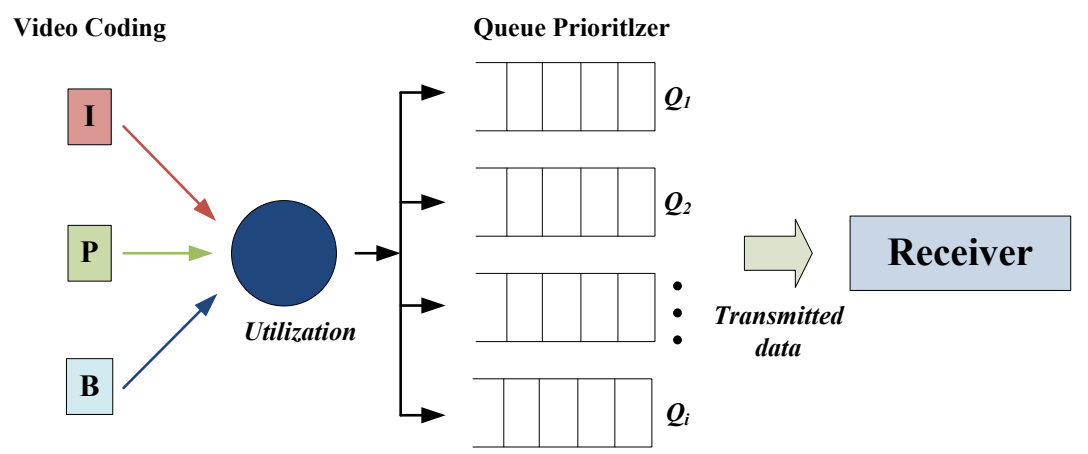

Fig.2. The queuing model.

In Fig. 2, consider a queuing model $Q_{i}$ with $M(i=1, \cdots, M-1)$ queues, and the weight coefficient $\omega_{i}$ for each queue $Q_{i}$. The queue utilization $\rho_{i}$ for queue $Q_{i}$ is defined as

$$
\rho_{i}=\frac{\bar{T}_{i}}{T} \times \omega_{i} .
$$

where $\bar{T}_{i}$ is the average transmission time of packet data if $T$ is the total transmission time of packet data, and is given by

$$
\bar{T}_{i}=\frac{1}{N} \sum_{j=1}^{N} \Delta t_{i, j}, \text { for } j=1,2, \cdots N
$$

where the inter-arrival time $\Delta t_{i, j}=t_{i, j}-t_{i, j-1}(\mathrm{~ms}) ; t_{i, j}$ and $t_{i, j-1}$ are timing (ms) when the receiver gets the $j^{\text {th }}$ and $(j-1)^{\text {th }}$ packets, respectively, for queue $Q_{i}$. The average utilization $\bar{\rho}$ of all queues is calculated by

$$
\bar{\rho}=\frac{1}{M} \sum_{i=0}^{M-1} \rho_{i}
$$

According to the average utilization $\bar{\rho}$ of all queues, the choice of available queue $Q_{i}$ for the video packet is based on when $\rho_{i}<\bar{\rho}$ and the video packet is mapped to the highest-priority queue $Q_{i}$ when $\rho_{i}<\bar{\rho}$, i.e., the $Q_{i}$ queue length is enough for sending the video packet. Otherwise, if $\rho_{i} \geq \bar{\rho}$, the video packet will be mapped to the other lower-priority $Q_{i}$ depending on which one is available in the sequence. Furthermore, the condition of dropping video packet when all queues $Q_{i}$ are not available or queue full.

Finally, let $D_{i}$ be the effective delay of queue $Q_{i}$, and $D_{i}^{0}$ be the idle delay of queue $Q_{i}$, the $D_{i}$ can be given by

$$
D_{i}=\frac{D_{i}^{0}}{1-\rho_{i}}
$$


When a queue $Q_{i}$ is idle, then the queue utilization $\rho_{i}=0$, and $D_{i}=D_{i}^{0}$; when the queue utilization $\rho_{i}$ is becoming bigger, then $D_{i}$ will be longer. In this paper, the queue utilization $\rho_{i}$ is used to determine the video packet is sent or dropped.

Proposed Cross-layer (SVC-Cross) Architecture. The procedure in each layer of the proposed cross-layer (SVC-Cross) architecture is shown in Fig. 3. While transmitting video packet, the APP layer could slice the original video frame using the H.264/SVC with the hierarchical B-picture approach [3][13] and classify the packet type by the use of packet classifier. In WiMAX, the proposed method avoids dropping massive packets in congested condition and keeping the queue availability (lowering its utilization) all the time, the MAC layer uses the packet priority and queue allocation approaches to improve the video bit-stream quality and transmission performance. The procedure to transmit the video packet shown in Fig. 3 is defined as follows:

- Step 1: Used the H.264/SVC with hierarchical B-picture approach [2]-[3][13], as shown in Fig.3, for APP layer. Scalable video packets (I, P, $B_{0}$, and $B_{1}$ ) are classified into the corresponding four packet types (Type A, B, C, and D) by the packet classifier at APP layer.

- Step 2: Video packets with four packet types (Type A, B, C, and D) are packed into the corresponding four slice types $\left(\right.$ Slice $_{\mathrm{A}}$, Slice $_{\mathrm{B}}$, Slice $_{\mathrm{C}}$, and Slice $\mathrm{D}$ ) based on the packet priority at the MAC layer.

- Step 3: The MAC layer performs the dynamic queue allocation using the queue utilization $\rho_{i}$, given in Eqs. (2)-(4), to improve the video bit-stream quality and transmission performance for the appropriate video packet.

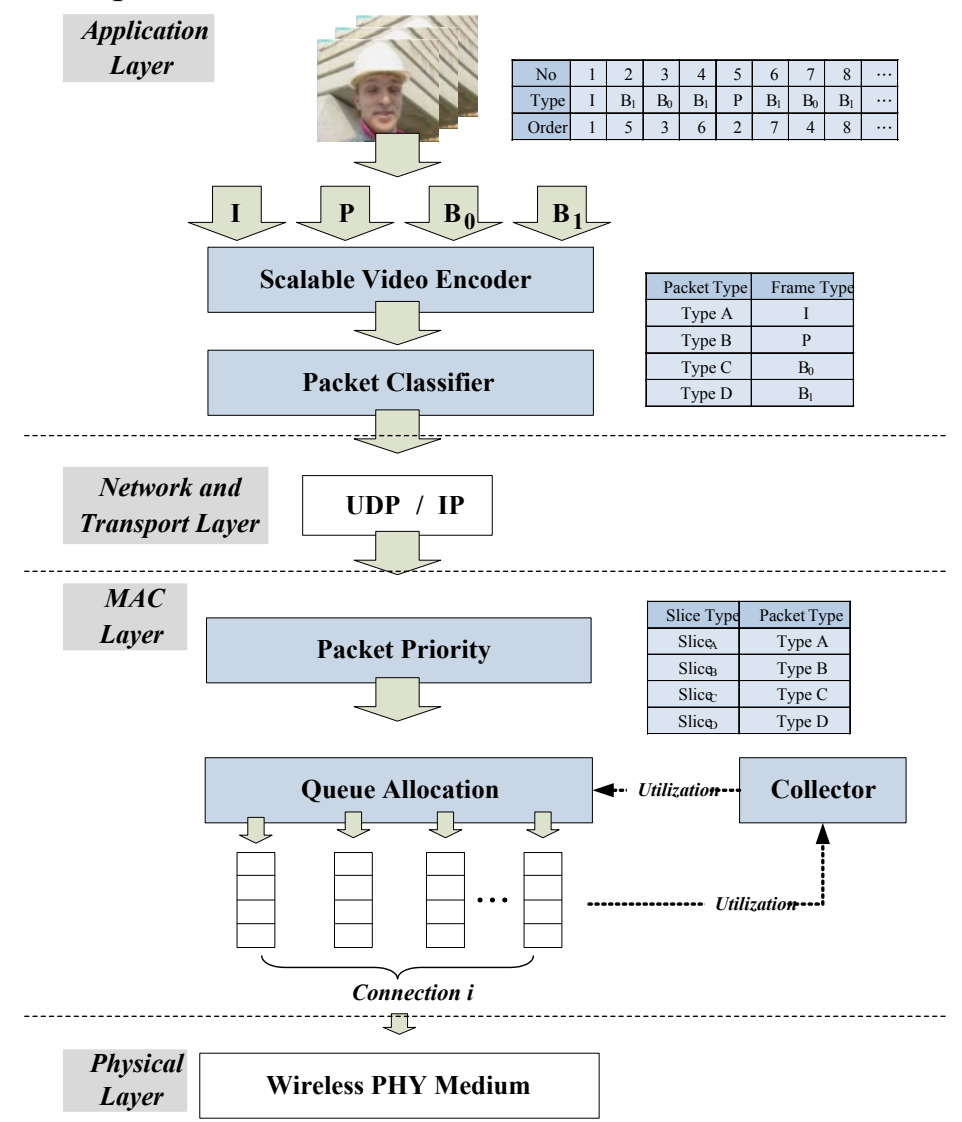

Fig.3. The proposed cross-layer (SVC-Cross) architecture.

\section{Performance Evaluation}

The cross-layer optimization architecture for WiMAX transmission proposed in this paper is to make the adjusting of transmit rate in the transmitter end as fast as possible, and to decrease the congestion effect during transmission, which improves the overall utilization of the network resources. We select the H.264/SVC algorithm, which JSVM 9.15 (Joint Scalable Video Model) is used, as the basis of comparison with experiment results. Some observed criterions include packet 
delay, jitter and rate-distortion (R-D) curves, etc. The simulated network is based on the NS2 2.29 (Network Simulator-version 2) [4] for WiMAX environment. The encoding GOP type of the SVC algorithm is IBBBPBBB... and the size of a GOP is 8 frames. In these experiments, we establish the UDP flow and observe the transmission processes by each case of the standard CIF video sequences (Foreman, News, and Coastguard).

Table 1. The bit rate and PSNR for Foreman CIF in WiMAX.

\begin{tabular}{|c|c|c|c|}
\hline \multirow{2}{*}{ Index } & \multicolumn{3}{|c|}{ PSNR (dB) } \\
\cline { 2 - 4 } & SVC-Cross & SVC-B3 & SVC-Quality \\
\hline 100 & 33.92 & 33.74 & 33.74 \\
\hline 500 & 35.73 & 35.49 & 35.65 \\
\hline 1000 & 37.22 & 37.43 & 37.58 \\
\hline 1500 & 39.14 & 38.73 & 38.66 \\
\hline 2000 & 40.02 & 39.99 & 39.99 \\
\hline
\end{tabular}

Table 2. The transmission delay for Foreman CIF in WiMAX.

\begin{tabular}{|c|c|c|}
\hline & Delay Time (ms) \\
\hline SVC-Cross & SVC-B3 & SVC-Quality \\
\hline Avg $: 18.87$ & Avg $: 21.18$ & Avg $: 22.56$ \\
Max $: 48.87$ & Max $: 58.53$ & Max $: 62.07$ \\
\hline
\end{tabular}

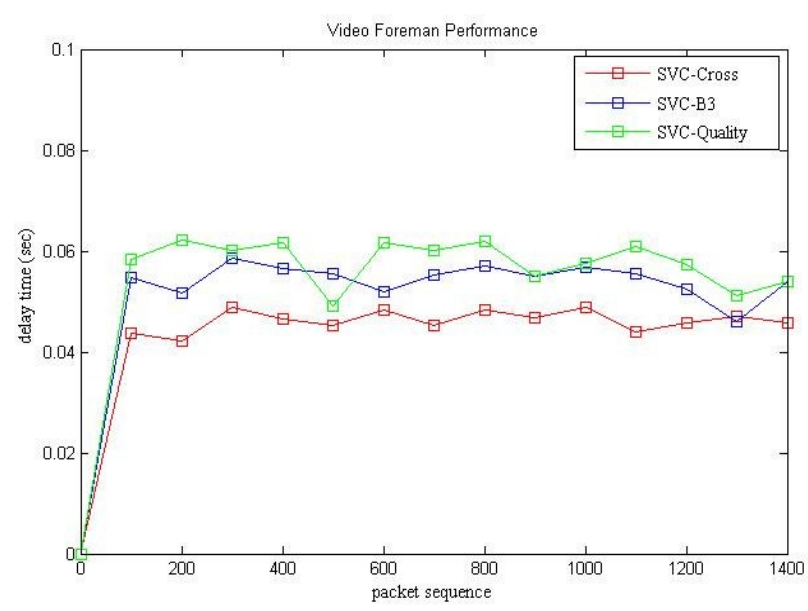

(a)

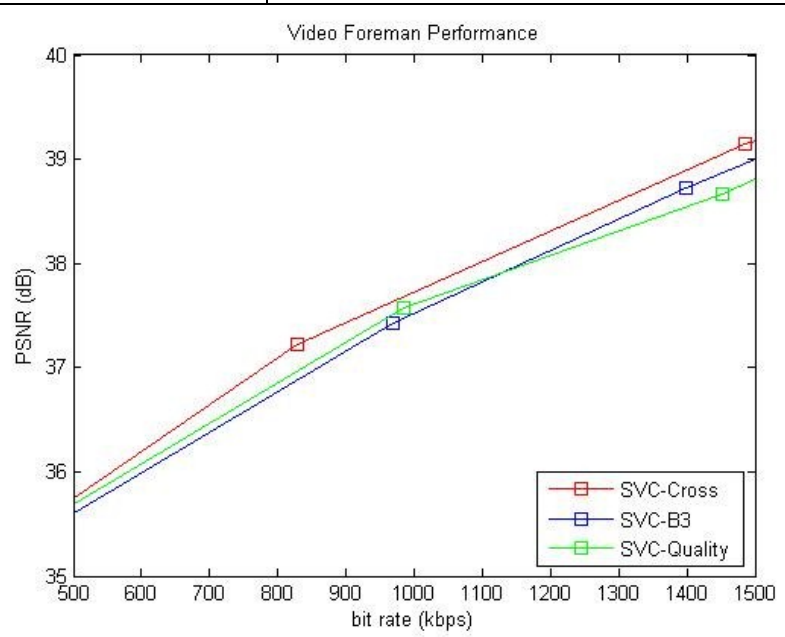

(b)

Fig.4. The (a) transmission delay and (b) R-D curve for Foreman CIF in WiMAX.

The experiment composes of several simulated video transmissions to evaluate the performance. We study the average delay time, packet jitter and video quality within the above three video transmissions: Foreman, News, and Coastguard, compared with the proposed SVC-Cross, the standard SVC-B3, and the standard SVC-Quality approaches. For brevity, only the experiment results of Foreman CIF sequence are shown in this section as an example. Table 1 shows the bit rate and PSNR for Foreman CIF sequence. In this table, the PSNR values obtained by the proposed SVC-Cross method are higher the standard SVC-B3 and SVC-Quality methods by about $0.5 \mathrm{~dB}$ at index 1500 for Foreman CIF sequence. That is, the proposed cross-layer method obtains a better visual quality of the reconstructed frames.

Table 2 shows the transmission delay time for Foreman CIF sequence. In this table, the average and maximum transmission delay time obtained by the proposed SVC-Cross method are less than the standard SVC-B3 and SVC-Quality methods for Foreman CIF sequence. Furthermore, the transmission delay and R-D curve for Foreman CIF sequence in WiMAX is shown in Fig. 4. In Fig. 4(a), the transmission delay obtained by the proposed SVC-Cross method is still less than the standard SVC-B3 and SVC-Quality methods. Also, one observation that the transmission delay is improved at least 50\% in the proposed cross-layer architecture. In Fig. 4(b), we can see the R-D curve for Foreman CIF sequence, the proposed SVC-Cross method can achieve a higher PSNR (dB) and is still better than the standard SVC-B3 and SVC-Quality methods. In other words, the proposed cross-layer method has a much better performance. 


\section{Summary}

In this paper, we construct a new cross-layer optimization to provide a better video delivery quality and to handle the issue of network congestion which affects severely the stability of wireless transmission based on the WiMAX network. We use the H.264/SVC with the hierarchical B-picture approach and the cross-layer architecture which uses the packet data classification and an adaptive queue allocation between APP and MAC layers. The simulation experiment proves that, the proposed cross-layer architecture can be constructed on the WiMAX network, the method which uses the scalable video encoder and packet classifier at APP layer and the packet priority and queue allocation at MAC layer does not consume any network resource. By the simulation results, the average transmission delay is improved at least $50 \%$ in the proposed cross-layer architecture, and getting a better quality of video bit-streams by adapting the transmission rate using queue utilization.

\section{Acknowledgment}

This work was supported by the Ministry of Science and Technology, R.O.C., under Grant MOST 104-2221-E-153-010.

\section{References}

[1] A. S. Abdallah and A. B. MacKenzie, A cross-layer controller for adaptive video streaming over IEEE 802.11 networks, in Proc. of IEEE International Conference on Communications, London, 2015, pp.6797-6802.

[2] H. Schwarz, D. Marpe, and T. Wiegand, Hierarchical B pictures, Joint Video Team, Doc. JVT-P014, 2005.

[3] H. Schwarz, D. Marpe, and T. Wiegand, Overview of the scalable video coding extension of the H.264/AVC standard, IEEE Transactions on Circuits and System for Video Technology, 17 (2007) 1103-1129.

[4] http://140.116.164.80/ smallko/ns2/svc.htm

[5] J. She, X. Yu, P. Ho, and E. Yang, A cross-layer design frame for robust IPTV services over IEEE 802.16 networks, IEEE Journal on Selected Areas in Communications, 27 (2009) $235-245$.

[6] J. Tian, H. Zhang, D. Wu and D. Yuan, Interference-Aware Cross-Layer Design for Distributed Video Transmission in Wireless Networks, IEEE Transactions on Circuits and Systems for Video Technology, 26 (2016) 978-991.

[7] K. Patil, Narayan D. G, U. Mudenagudi and J. Amboji, Cross layer routing and rate adaptation for video transmission in multi-radio Wireless Mesh Networks, in Proc. of International Conference on Advances in Computing, Communications and Informatics, Kochi, 2015, pp.2139-2144.

[8] L. Harte, Introduction to 802.16 WiMAX, Althos Publishing, 2006.

[9] L. Nuaymi, WiMAX : Technology for Broadband Wireless Access, Wiley, 2007.

[10] L. J. Wang and C. E. Wu, Cross-layer architecture for video transmission over wireless multimedia networks, in Proc. of Mosharaka International Conference on Wireless Communications and Mobile Computing, Istanbul, Turkey, 2011, pp. 3-5.

[11] L. J. Wang, C. E. Wu, C. Y. Chang, A cross-layer architecture with service adaptability for wireless multimedia networks, International Journal of Simulation- Systems, Science and Technology, 16 (2015) 2.1-2.9. 
[12] M. V. der Schaar and S. Shankar, Cross-layer wireless multimedia transmission: challenges, principles, and new paradigms, IEEE Wireless Communications, 12 (2005) 50-58.

[13] M. Li, Z. Chen, and Y. P. Tan, Cross-layer optimization for SVC video delivery over the IEEE 802.11e wireless networks, Journal of Visual Communication and Image Representation, 22 (2011) 284-296.

[14] M. Zhao, X. Gong, J. Liang, W. Wang, X. Que and S. Cheng, QoE-Driven Cross-Layer Optimization for Wireless Dynamic Adaptive Streaming of Scalable Videos Over HTTP, IEEE Transactions on Circuits and Systems for Video Technology, 25 (2015) 451-465.

[15] S. Milani and G. Calvagno, A low-complexity cross-layer optimization algorithm for video communication over wireless networks, IEEE Transactions on Multimedia, 11 (2009) 810-821.

[16] Y. T. Mai, C. C. Yang, and Y. H. Lin, Design of the cross-layer QoS framework for the IEEE 802. 16 PMP networks, IEICE Transactions on Communications, E91- B (2008) 1360-1369. 